\title{
L'apprentissage des notions mathématiques chez des élèves ayant une déficience intellectuelle légère entre une approche pédagogique traditionnelle et la médiation artistique
}

\section{The learning of mathematical concepts in students with a mild intellectual disability between a traditional pedagogical approach and artistic mediation}

\author{
Salem Saaidia ${ }^{1 *}$ and Faten El Meddah ${ }^{2}$ \\ ${ }^{1}$ Etudiant mastère, Institut Supérieur de l'Education Spécialisée (ISES), Université de la Manouba, \\ Tunisie. \\ ${ }^{2}$ Maître assistant, Institut Supérieur de l’Education Spécialisée (ISES), Université de la Manouba, \\ Tunisie.
}

\begin{abstract}
Résumé. Cette étude examine l'apport des médiations artistiques à l'acquisition des notions et des habiletés mathématiques chez des élèves ayant une déficience intellectuelle légère, âgés entre 11 et 13 ans et poursuivant une formation professionnelle en pâtisserie. Afin de mettre en exergue l'apport de cette médiation, nous avons travaillé avec un échantillon expérimental composé de 8 élèves et un échantillon témoin et nous avons comparé leurs résultats. Nous avons pu montrer à travers notre intervention que le recours à la médiation artistique ainsi qu'à l'apprentissage par la découverte pourrait mieux aider les élèves à maîtriser les notions et les habilités mathématiques utiles dans la vie quotidienne et professionnelle, dans le cas des enfants ayant une déficience intellectuelle légère.
\end{abstract}

\begin{abstract}
This research examines the contribution of artistic mediations on the acquisition of mathematical concepts and skills among students with mild intellectual disabilities, aged between 11 and 13 years old and pursuing professional training in pastry making. We worked with an experimental sample of 8 students and another control sample to make sure in illuminating comparison is possible. We were able to show through our intervention that the use of artistic mediation could better help students master mathematical concepts and skills useful in daily and professional life and that learning
\end{abstract}

\footnotetext{
* Corresponding author: salem.saaidia@ises.u-manouba.tn
} 
through discovery would also improve the acquisition of mathematical concepts and skills, in the case of children with mild intellectual disabilities.

\section{Introduction et problématique}

Depuis plusieurs années, la spécificité de l'enseignement des mathématiques aux élèves présentant une déficience intellectuelle a fait une question vive et un grand défi pour les décideurs des programmes, les institutions scolaires, les éducateurs mais aussi pour ces élèves $[5,11,15,1,4,47]$. Dans ce cadre, plusieurs chercheurs suggèrent que l'enseignement des mathématiques dans un contexte d'adaptation scolaire notamment pour les élèves déficients intellectuels représente un grand défi pédagogique mais aussi didactique. En fait, les enseignants et les éducateurs trouvent des difficultés pour mettre en place des situations didactiques afin de permettre à ces élèves de s'approprier les objets du savoir mathématique $[2,17,18,19]$. Par ailleurs, il est important sur le plan pédagogique d'encourager les élèves à la manipulation d'une large variété de matériel concret ainsi qu'à l'utilisation de représentations visuelles, schémas et dessins pour explorer les différentes solutions possibles [20].

Sur le plan didactique, la question de l'accessibilité aux savoirs mathématiques pour les élèves avec une déficience intellectuelle reste une énigme peu traitée. En d'autres termes, la spécificité de l'intervention didactique auprès des élèves en difficulté réside dans les conditions et les situations didactiques à mettre en œuvre afin de garantir une prise en charge multi-dimensionnelle de l'élève, l'individualisation des apprentissages et un accompagnement socio-éducatif personnalisé pour à leur niveau intellectuel et à leurs possibilités singulières [8].

Par ailleurs, malgré les dispositifs mises en œuvre en faveur des personnes en situation de handicap en général et ceux ayant une déficience intellectuelle, la situation de leur intégration professionnelle reste problématique. "La difficulté d'intégration dans le monde du travail serait en fait très liée à des facteurs relationnels et à la présence de comportements problématiques souvent liés à un déficit des comportements adaptatifs » [21]. Dans ce cadre l'auteur propose trois pistes pour pouvoir développer les compétences adaptatives chez les personnes ayant une déficience intellectuelle: la mise en place d'un accompagnement humain adapté à leurs besoins, concevoir des outils pour contourner leur difficulté et travailler les capacités cognitives impliquées [21].

L'enseignement des mathématiques aux élèves ayant une déficience intellectuelle représente une grande problématique dans le contexte de l'éducation spécialisée pour deux raisons : d'une part, les éducateurs font recours à des méthodes qui ne stimulent pas la motivation de ces élèves et qui ne respectent pas leur développement intellectuel et social [43]. D'autre, la question de l'utilité des apprentissages à dispenser à ces élèves ainsi que les objectifs de l'enseignement spécialisé qui font un dilemme quel que soit sur le plan pédagogique ou didactique [23].

Dans le présent travail, nous avons adopté une démarche pédagogique basée sur l'apprentissage par la découverte qui consiste en un mode d'apprentissage par la pratique et la mobilisation des connaissances dans des situations de la vie quotidienne en manipulant des objets concrètes et ceci à travers l'utilisation de la médiation artistique. Ainsi, nous avons conçu et mis à l'essai un programme d'intervention éducative afin d'examiner l'apport de trois médiations artistiques à savoir la médiation musicale, la médiation théâtrale et celle par les arts-plastiques pour l'acquisition de quelques habilités et notions mathématiques utiles dans la vie professionnelle telles que gérer la monnaie, le temps, les unités de mesure de la longueur (le mètre) et de poids (kilogramme) et la géométrie dont le dessin des formes géométriques (carrée, triangle et cercle) et l'utilisation de l'unité de centimètre chez des 
élèves ayant une déficience intellectuelle légère âgés entre 11 et 13 ans et qui poursuivent une formation professionnelle en pâtisserie.

Faisant référence à ce que nous venons de présenter, nous posons les questions suivantes :

1. Quel est l'apport de l'utilisation de la médiation artistique (médiation théâtrale, médiation musicale et celle par les arts-plastiques) sur l'apprentissage des notions et des habilités mathématiques chez des élèves ayant une déficience intellectuelle légère?

2. Quel est le rôle de l'apprentissage par la découverte dans l'acquisition des notions et des habilités mathématiques chez des élèves ayant une déficience intellectuelle légère?

3. Y-a-t-il de différence entre les médiations artistiques utilisées pour l'apprentissage des notions et des habilités mathématiques chez des élèves ayant une déficience intellectuelle légère?

4. Quelle est la différence entre l'utilisation de la médiation artistique et celle d'une approche pédagogique traditionnelle sur l'acquisition des notions et des habilités mathématiques chez des élèves ayant une déficience intellectuelle légère?

\section{Cadre théorique}

Dans cette partie nous présentons les concepts de base de ce travail

\subsection{La déficience intellectuelle et l'apprentissage des mathématiques}

De point de vue cognitive et développementale, la déficience intellectuelle s'exprime par un double constat : le premier est en relation avec la différence c'est-à-dire moindre efficience par rapport à l'âge chronologique et le deuxième consiste en un retard par rapport aux normes psychométriques déterminées à travers des échelles d'intelligence [16, 22]. Dans son rapport d'expertise, l'INSERM propose la définition suivante de la déficience intellectuelle : Elle " [...] fait référence à un déficit de l'intelligence ainsi qu'à un déficit du comportement adaptatif qui y est associé. C'est le terme le plus couramment utilisé actuellement dans la littérature internationale dans le champ de la médecine, de l'éducation ou de la psychologie » [13].

Le DSM 5 [12] caractérise la déficience intellectuelle légère par des difficultés dans les habilités conceptuels qui résident dans le recours à une manière plus pragmatique dans la résolution des problèmes. Pour les habilités sociales, les personnes avec une déficience intellectuelle légère montrent une compréhension limitée des risques dans des situations sociales. Enfin, pour les habilités pratiques, la personne manifeste des difficultés pour faire des tâches qui nécessitent beaucoup d'habilités conceptuelles.

La déficience intellectuelle en général et celle légère en particulier a plusieurs répercussions sur les apprentissages et leurs acquisitions. Dans ce cadre, trois domaines fondamentaux qui interviennent dans l'acquisition des apprentissages et qui sont touchées par la déficience intellectuelle sont distingués [13] : Tout d'abord, les théories cognitives postulent que les personnes ayant une déficience intellectuelle souffrent des difficultés au niveau de mémoire de travail, mémoire à court terme responsable du stockage des informations au cours de l'exécution d'une tâche donnée et de l'acquisition de nombreuses compétences comme la lecture, le langage, le calcul, le raisonnement. Plusieurs études ont montré que la mémoire de travail des personnes ayant une déficience intellectuelle est limité en comparaison avec celle des enfants au développement typique. Les fonctions exécutives qui sont classées en trois grandes fonctions : la mémoire de travail, l'inhibition et la flexibilité sont aussi fragiles chez les personnes déficientes intellectuels en comparaison avec des 
personnes ayant un développement typique. L'étude mené par l'INSERM a montré que les performances des personnes ayant une déficience intellectuelle dans le domaine des fonctions exécutives ne sont pas seulement liées au QI mais dépendent également du comportement adaptatif. Enfin, la déficience intellectuelle est liée directement à un développement retardé $d u$ langage. Le langage intervient dans la construction, l'acquisition et l'appropriation des apprentissages. Certes, "la déficience intellectuelle est une variable causale majeure des difficultés langagières classiquement observées chez les personnes avec déficience intellectuelle. Toutes les composantes du langage sont concernées (phonologique, lexicale, morphosyntaxique et pragmatique). " [13]. Il est à noter que l'acquisition des habilités et des notions mathématiques nécessite la mise en œuvre de ces fonctions cognitives [2].

Les enseignements destinés à ces élèves doivent être utilitaires. Le choix des objectifs éducatifs se fait aussi en fonction de leur utilité dans la vie quotidienne de l'élève. "Tout apprentissage mathématique devrait être choisi en fonction de son utilité pour la vie quotidienne et l'insertion professionnelle » [25]. En outre, l'objectif principal de l'enseignement pour les personnes déficients intellectuelles c'est d'améliorer leur quotidien [27]. Ainsi, les mathématiques sont impliquées dans de nombreuses activités de la vie quotidienne comme l'évaluation des quantités (longueurs, poids, volumes...), la mesure de la température et du temps (en utilisant des instruments de mesure) et la manipulation de l'argent. Plusieurs recherches [14, 11,4] ont ainsi démontré que les élèves ayant des incapacités intellectuelles légères avaient besoin d'une attention particulière et davantage de pratique pour acquérir des aptitudes dans cette discipline et d'être capables d'automatiser les différentes habiletés mathématiques de base, notamment le calcul, la numérotation et la représentation du problème.

Par ailleurs, l'acte d'apprendre en général et l'apprentissage des mathématiques en particulier chez les élèves ayant une déficience intellectuelle nécessite une attention particulière sur le processus enseignement/apprentissage afin d'éviter les obstacles pédagogiques ou environnementaux qui peuvent empêcher le processus de leur apprentissage. Pour cela il est important d'avoir des aménagements particuliers pour favoriser leur apprentissage $[26,25,30,20]$. En d'autres termes, les principes généraux de l'intervention avec les personnes ayant une déficience intellectuelle sont : tout d'abord, une intervention efficace se base sur l'adaptation des méthodes d'intervention en fonction de l'âge intellectuel que chronologique. Ensuite, la généralisation des apprentissages qui est une tâche très difficile chez les personnes présentant une déficience intellectuelle. Pour cela "l'intervenant devrait cibler des apprentissages concrets et fonctionnels, c'est-à-dire des interventions qui auront un impact sur le fonctionnement et l'autonomie de la personne " [20]. Enfin, la question de durée et de fréquence des activités proposées se pose dans un contexte d'accompagnement socio-éducatif des personnes avec une déficience intellectuelle puisqu'ils sont moins attentifs, c'est pour cette raison il est nécessaire d'avoir une plus grande fréquence d'intervention et de répétition des activités pour avoir des meilleurs résultats.

\subsection{La médiation artistique}

Les pratiques de la médiation artistique ont été développées depuis longtemps dans la clinique psychiatrique comme une forme de thérapie psychanalytique [31]. Aujourd'hui, cette médiation est pratiquée dans plusieurs secteurs de l'accompagnement éducatif et social notamment avec les personnes en difficultés afin de les assister et les aider dans leurs processus d'apprentissage et de création des liens sociaux [32, 33]. La médiation artistique est définie ainsi comme l'utilisation de l'art et de la création artistique pour des visées d'accompagnement socio-éducatif mais aussi pour stimuler les facultés d'expression et dynamiser les processus créatifs de la personne [34]. Dans le secteur du travail social, il s'agit d'une médiation éducative [46]. «La médiation artistique peut aider à faire renaître un désir, 
quel qu'il soit : apprendre, se réinscrire dans le groupe social, refonder un lien social distendu, dégager de nouvelles pistes pour faire face à un quotidien devenu insupportable » [29]. Certes, l'art consiste dans ce sens en une forme d'accessibilité pour les personnes en situation de handicap. En fait, les prises en charge par le biais de l'art peuvent offrir les conditions et les opportunités d'accès à des pratiques qui favorisent l'orientation des personnes vers des activités (musique, théâtre, arts-plastiques, etc.) qui enrichiront leur quotidien et pourront concourir à son mieux-être [34].

La médiation musicale consiste, pour le présent travail, en l'utilisation de la musique pour des visées éducatives. Il y a deux formes de musique : la première forme est active dont sa pratique privilège la production sonore et musicale, l'improvisation, la créativité. La deuxième forme est dite réceptive puisqu'elle propose des dispositifs fondés sur l'écoute [35].

\subsection{L'apprentissage par la découverte}

L'apprentissage par la découverte est le modèle d'enseignement inspiré du courant cognitiviste et nous nous intéressons au modèle proposé par Bruner [36]. L'apprentissage par la découverte est un modèle d'enseignement-apprentissage socioconstructiviste d'inspiration cognitiviste selon la perspective de Bruner [37]. Il s'agit de "l'enseignement d'une association, d'un concept ou d'une règle impliquant la découverte de cette association, de ce concept ou de cette règle " [38]. Ce modèle considère l'apprentissage comme un processus actif centré sur la manipulation et la découverte dont l'élève est un acteur responsable de ses apprentissages [39].

L'apprentissage par la découverte est considéré comme moyen qui suppose l'apprentissage à découvrir comme objectif. En d'autres termes, l'apprentissage par la découverte amène l'élève dans des situations d'apprentissage motivantes et bien structurées de découvrir et de construire ses apprentissages afin de lui permettre de mobiliser ses acquisitions dans des situations diverses. Donc ce modèle est un moyen d'apprentissage qui développe le désir d'apprendre dans un environnement stimulant [37].

On distingue six principes de l'apprentissage par la découverte [37]: Tout d'abord, l'enseignant doit adopter une attitude d'animateur, de médiateur ou d'accompagnateur afin d'offrir les conditions favorables pour l'apprentissage de ses élèves en créant des situations d'apprentissage qui stimulent leur curiosité d'apprentissage, leur motivation et leur exploration. Le renforcement est aussi l'un des principes de ce modèle où l'intervenant doit récompenser l'élève dans son processus d'apprentissage par la découverte notamment les récompenses de type intrinsèques comme les félicitations ou autres. En outre, l'enseignant doit assister l'élève pour avoir des compatibilités entre ses découvertes et les connaissances qu'il possède déjà pour les mettre en liens. L'entraînement est aussi un élément très important dans l'apprentissage par la découverte puisqu'il permet de structurer les apprentissages et de confronter d'autres à travers des hypothèses et des expérimentations. La découverte nécessite la maîtrise du langage ainsi qu'une prise de conscience de soi. L'élève développe sa conscience en soi et ses compétences linguistiques par le débat et la discussion avec l'enseignant ou avec ses pairs autour de la situation d'apprentissage et il développe aussi sa pensée réflexive. Enfin, "la démarche d'apprentissage par la découverte a pour but de développer des aptitudes à traiter l'ensemble des éléments d'information disponibles afin de s'en servir pour résoudre des problèmes de façon appropriée dans un contexte particulier » [37].

Pour les élèves ayant des besoins éducatifs particuliers dont ceux ayant une déficience intellectuelle légère, la démarche pédagogique doit s'articuler sur l'apprentissage par la découverte en offrant un enseignement pratique et significatif [24]. Par ailleurs, l'apprentissage n'est pas seulement une démarche mobilisée pour l'acquisition des 
compétences définies par l'éducateur, mais aussi une culture des stratégies cognitives de l'élève, un affinement de ses capacités et par conséquent, une méthode pour « apprendre à apprendre » [48]. En fait, l'apprentissage par la découverte aide les personnes ayant une déficience intellectuelle à mobiliser leurs connaissances dans des situations de la vie quotidienne et ceci leur permet de s'intégrer dans la société et d'être plus autonome dans leur vie.

\subsection{L’approche pédagogique traditionnelle}

Altet propose une classification des courants pédagogiques [40]. Cette classification décrit la pédagogie comme une régulation fonctionnelle et dialectique entre les processus « enseignerapprendre, apprendre-enseigner ». Elle a distingué quatre courants de ce modèle, le courant magistro-centriste basé sur la transmission des savoirs dont le Maître prépare le savoir, organise la situation et transmet le savoir aux élèves qui reçoivent le savoir. Ce courant est caractéristique des approches pédagogiques traditionnels et classiques. Le deuxième courant est celui puero-centriste dont l'apprentissage est une construction personnelle et son objectif principal est de développer et de former un élève responsable à ses apprentissages en donnant la priorité à ses intérêts. C'est le cas de la pédagogie active de Decroly. Le troisième courant est le courant socio-centriste qui est fondé sur la formation d'un homme social et de l'éduquer socialement, citons comme exemple la pédagogie Freinent. Enfin, contrairement aux autres courants, le courant centré sur l'apprentissage définit l'acte pédagogique du point de vue de l'élève. La pédagogie traditionnelle fait partie ainsi du courant magistro-centriste, elle est fondée sur l'utilisation d'un ensemble de méthodes et techniques pédagogiques classiques fondées sur la transmission des savoirs où l'éducateur est au centre de la situation pédagogique puisqu'il met et fait toutes les tâches sans prendre en compte les singularités et les potentialités de chaque élève [41, 42].

Par ailleurs, la majorité de contenus d'enseignements dispensés par les éducateurs spécialisés aux élèves déficients mentaux se caractérise par un recours à des séquences d'enseignement de type artistique cependant les enseignements de base comme la lecture, le calcul et l'écriture sont rarement dispenser aux élèves ayant une déficience intellectuelle. En outre, les durées des séquences d'apprentissage semblent ne pas être adaptées à ces enfants et elles ne respectent pas les potentialités et les singularités de chaque élève. L'auteure montre ainsi que les éducateurs spécialisés négligent les apprentissages de base dans leurs pratiques d'intervention et ils font recours à des méthodes qui ne stimulent pas la motivation d'apprentissage chez leurs élèves [43].

\section{Approche méthodologique}

L'objectif général de cette recherche est d'étudier l'apport de l'utilisation des médiations artistiques sur l'apprentissage des notions et des habilités mathématiques aux élèves ayant une déficience intellectuelle légère. Nous présentons ainsi dans cette partie notre approche méthodologique.

\subsection{L'échantillon}

Pour des visées comparatives entre l'effet des deux méthodes (la méditation artistique et l'approche pédagogique traditionnelle) sur l'apprentissage de quelques notions et habilités mathématiques chez les élèves ayant une déficience intellectuelle légère, nous avons sélectionné deux échantillons portant les mêmes caractéristiques (déficience intellectuelle légère et âge biologique entre 11 et 13 ans, les élèves poursuivent une formation 
professionnelle en pâtisserie). Ces deux échantillons sont choisis dans l'unité de l'éducation spécialisée et de la réhabilitation professionnelle de l'institut supérieur de l'éducation spécialisée « la volonté » de la Manouba, Tunisie. Il est à signaler que les élèves sont diagnostiqués par le psychologue de l'institution comme porteurs d'une déficience intellectuelle légère et ceci est noté dans leurs dossiers psycho-éducatifs.

Le premier échantillon est expérimental et composé de 8 élèves ayant une déficience intellectuelle légère, ils ont suivi un enseignement des mathématiques par le biais de trois médiations artistiques (la musique, le théâtre et les arts-plastiques). Ces élèves poursuivent une formation professionnelle en pâtisserie afin d'obtenir un diplôme qui leur permet de s'intégrer dans la vie professionnelle après.

Le deuxième échantillon est témoin et composé de 7 élèves ayant aussi une déficience intellectuelle légère et poursuivent aussi une formation professionnelle en pâtisserie. Ces élèves ont bénéficié d'un enseignement des mathématiques par le bais d'une approche pédagogique traditionnelle.

\subsection{Le test}

Pour évaluer leurs acquisitions en mathématiques, nous avons fait recours à un test d'évaluation. Ce test est élaboré en se focalisant sur quatre notions et habilités mathématiques qui font partie de leur programme d'enseignement/apprentissage à savoir la manipulation de l'argent, l'évaluation des quantités dont les masses et les longueurs, la mesure du temps et enfin la géométrie et l'utilisation de l'unité de centimètre.

Les questions sont élaborées en fonction des situations réparties en 10 questions :

\section{Pour la manipulation de l'argent :}

Pour la première consigne il s'agit de compléter la pièce de monnaie manquante pour avoir la somme demandée. Et Pour la deuxième consigne, c'est une situation de la vie quotidienne où les élèves doivent calculer la somme des pièces de monnaie.

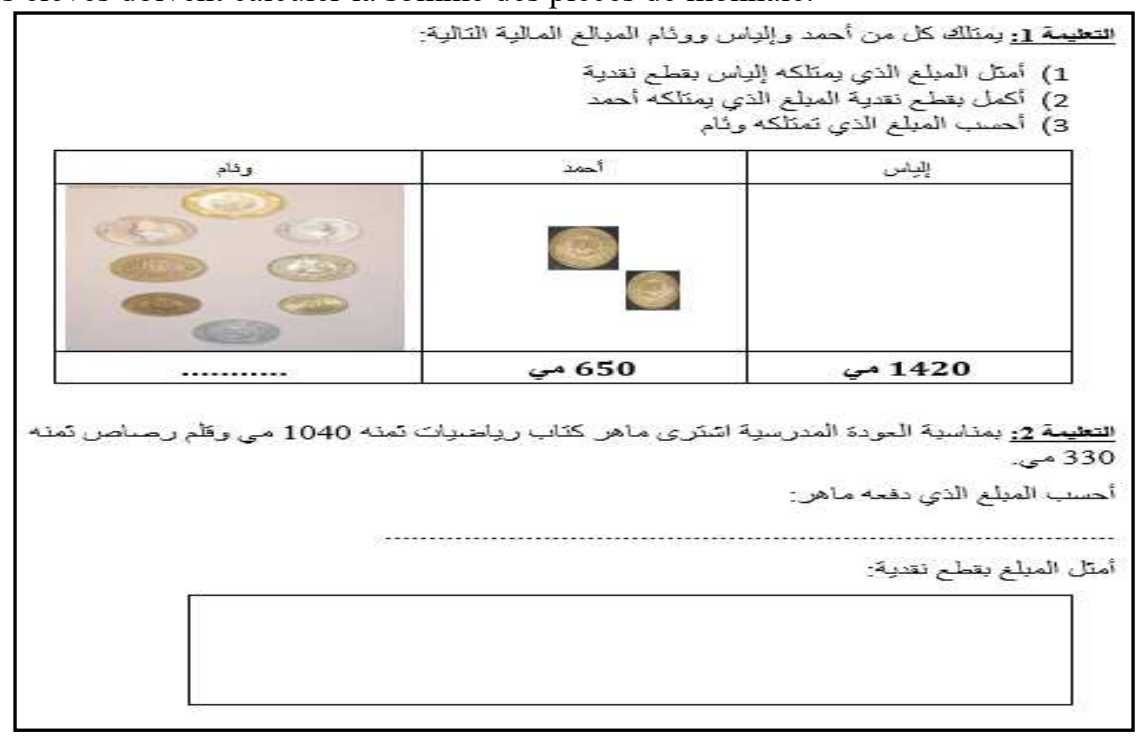

Fig. 1. Les consignes du test pour évaluer la notion « manipulation de l'argent ».

\section{Pour l'évaluation des quantités :}

Nous demandons aux élèves de relier chaque objet par son unité de mesure. 


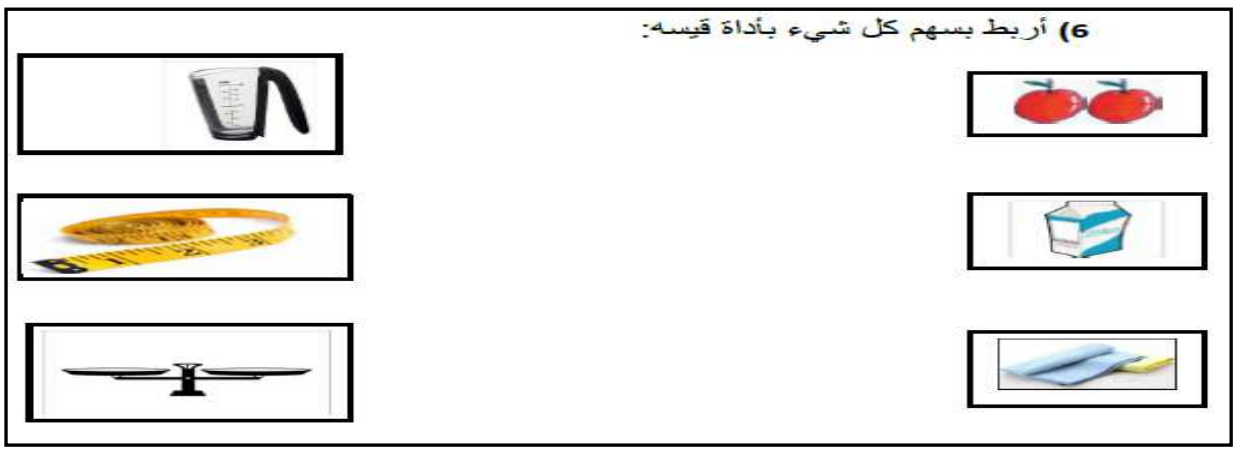

Fig. 2. La consigne du test pour évaluer la notion "l'évaluation des quantités».

\section{Pour la mesure du temps :}

Pour évaluer leurs acquisitions dans la mesure de temps, les élèves ont répondu à deux questions : la première consiste à lire le temps sur la montre et l'écrire. Et la deuxième consiste à représenter le temps sur une montre.
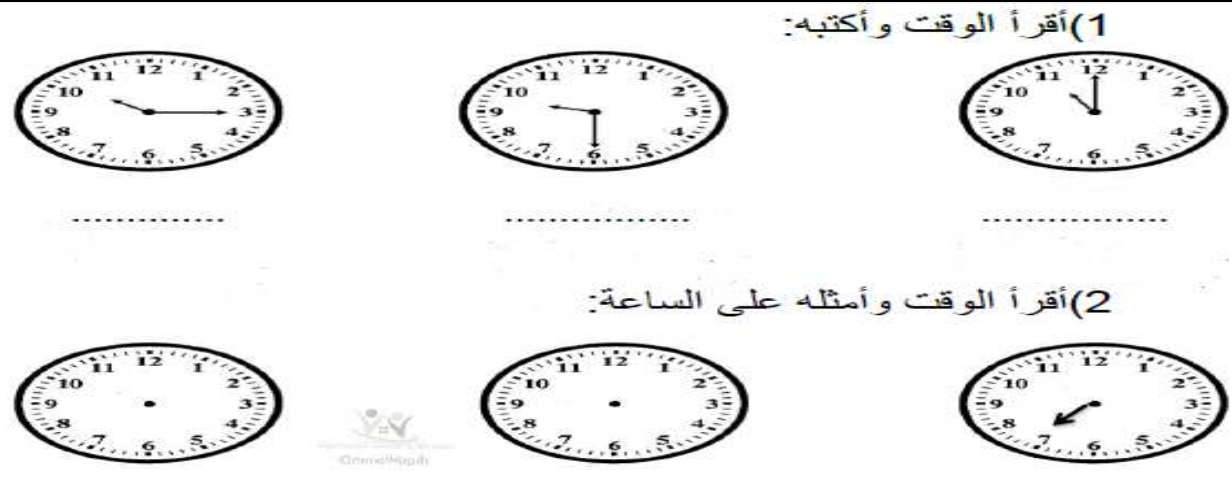

$: 15$

$12: 30$

$07: 10$

Fig. 3. Les consigne du test pour évaluer la notion « mesure du temps».

\section{Pour la géométrie et l'unité de "centimètre "}

Pour la géométrie et l'unité de centimètre, dans un premier temps, les élèves doivent dessiner une ligne droite de $12 \mathrm{~cm}$ de longueur et ils doivent dessiner un triangle, une carrée et un cercle dans un deuxième temps.

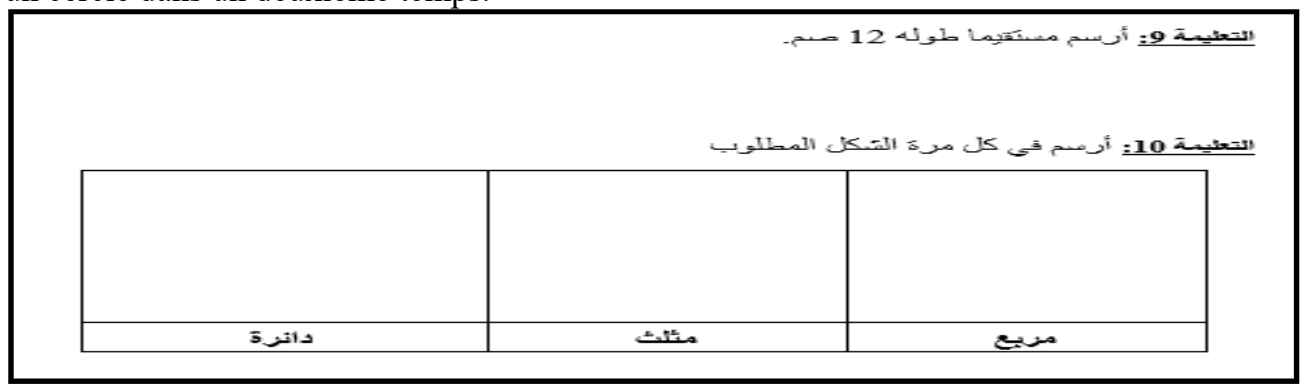

Fig. 4. Les consigne du test pour évaluer la notion " géométrie et l'unité de centimètre».

Pour la mise en place de ce test, nous avons pris en considération les potentialités et les singularités des élèves des deux échantillons. 


\subsection{Déroulement de l'expérimentation}

Nous avons conçu et mis à l'essai un programme d'intervention éducative spécialisé dont les activités sont basées sur l'utilisation de la musique, le théâtre et les arts-plastiques. Ces activités sont élaborées en se référant aux capacités cognitives et intellectuelles chez les élèves de notre échantillon expérimental ainsi que la théorie des intelligences multiples dont l'intelligence kinésique et musicale.

L'expérimentation s'est déroulée pendant 7 semaines, au rythme de trois interventions par semaine. Le tableau suivant présente le programme d'intervention éducative spécialisée destiné à notre échantillon expérimental :

Tableau 1 : le programme d'intervention éducative spécialisée

\begin{tabular}{|c|c|c|}
\hline $\begin{array}{l}\text { Notion et habilité } \\
\text { mathématiques }\end{array}$ & Médiations utilisées & Objectifs pédagogiques \\
\hline $\begin{array}{l}\text { La mesure du temps } \\
(M T)\end{array}$ & $\begin{array}{l}\text { La médiation par les } \\
\text { arts-plastiques } \\
\text { La médiation } \\
\text { théâtrale } \\
* \quad \text { La médiation } \\
\text { musicale }\end{array}$ & $\begin{array}{l}\text { Distinguer entre les aiguilles de } \\
\text { la montre. } \\
\text { Lire le temps. } \\
\text { Distinguer entre les minutes et } \\
\text { les heures. }\end{array}$ \\
\hline $\begin{array}{c}\text { La manipulation de } \\
\text { l'argent }(M A)\end{array}$ & $\begin{array}{l}\text { La médiation par les } \\
\text { arts-plastiques } \\
\text { La médiation } \\
\text { théâtrale }\end{array}$ & $\begin{array}{l}\text { Distinguer entre les pièces de } \\
\text { monnaie entre } 5 \text { millimes et } 5 \\
\text { dinars. } \\
\text { Manipuler l'argent dans des } \\
\text { situations de la vie quotidienne. }\end{array}$ \\
\hline $\begin{array}{l}\text { L'évaluation des } \\
\text { quantités }(E Q) \text { : } \\
\text { Les poids ; } \\
\text { Les longueurs ; }\end{array}$ & $\begin{array}{l}\text { La médiation } \\
\text { théâtrale }\end{array}$ & $\begin{array}{l}\text { Utiliser les outils et les unités } \\
\text { de mesure des poids. } \\
\text { Utiliser les outils et les unités } \\
\text { de mesure des longueurs. }\end{array}$ \\
\hline $\begin{array}{c}\text { La géométrie et } \\
\text { l'unité de mesure : le } \\
\text { centimètre } \\
\text { (Géométrie) }\end{array}$ & $\begin{array}{l}\text { La médiation par les } \\
\text { arts-plastiques } \\
\text { La médiation } \\
\text { musicale }\end{array}$ & $\begin{array}{l}\text { Dessiner des formes } \\
\text { géométriques : cercle, carrée et } \\
\text { triangle. } \\
\text { Distinguer entre les distances. }\end{array}$ \\
\hline
\end{tabular}

Il est à noter que chaque activité se termine par une séance de débats entre les élèves afin de leur permettre de réfléchir et d'évaluer les différentes étapes de la démarche de l'activité. Nous avons essayé aussi d'accompagner les élèves dans un processus de réflexion sur leurs apprentissages. Ces séances de débats nous ont permis de suivre les apprentissages des élèves. Dans ce qui suit, nous allons décrire les activités et présenter les résultats

\section{Résultats et discussion}

Dans cette partie nous allons présenter les résultats obtenus après la mise en œuvre de notre programme d'intervention éducative spécialisée tout en comparant entre les résultats du post et du pré-test. Les résultats et leur discussion seront présentés en fonction de la médiation artistique utilisée. 


\subsection{La médiation par les arts-plastiques... une forme d'apprentissage par le concret}

Les résultats du pré-test montrent que les élèves des deux échantillons ont des difficultés pour distinguer entre les tailles des aiguilles de la montre. En fait, les élèves n'arrivent pas à représenter les aiguilles sur la montre et certains d'entre eux ne distinguent pas entre l'aiguille des heures et celle des minutes.

Par conséquent, nous mettons en œuvre une activité d'arts-plastiques dont l'objectif est de fabriquer un modèle d'une montre en utilisant les abaisses langues (pour fabriquer les aiguilles) et les coquilles (pour indiquer les numéros de la montre) suivie d'une activité de coloriage (fig. 5). Après cette activité, les élèves arrivent à distinguer et à représenter correctement les aiguilles de la montre selon l'heure indiquée. Ceci n'est pas le cas des élèves de l'échantillon témoin qui montrent aussi, même après l'intervention par le biais d'une approche pédagogique traditionnelle (fig.6), des difficultés pour représenter de façon correcte les aiguilles de la montre.

Fig. 5. Une montre réalisée par un apprenant.
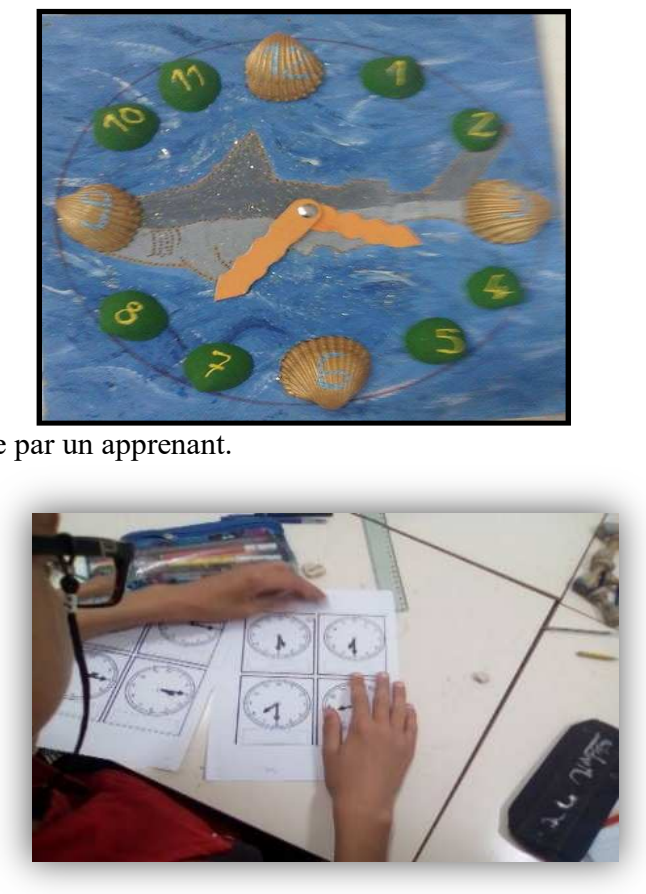

Fig. 6. Apprentissage de mesure du temps par l'ET.

En relation avec la manipulation de l'argent, les élèves des deux échantillons montrent des difficultés à distinguer entre les différentes pièces de monnaie ( 5 millièmes et 5 dinars), ainsi que des difficultés pour déterminer leurs valeurs (millièmes ou dinars). Suite à ces résultats, nous faisons recours à une activité de coloriage des pièces de monnaie en fonction de leur couleur réel et une autre activité pour dessiner les pièces de monnaie demandées. Les résultats du post-test nous montrent que les élèves arrivent à distinguer entre les différentes pièces de monnaie en fonction de leur taille et de leur couleur. Par contre, après l'intervention par le biais d'une approche pédagogique traditionnelle, les élèves de l'échantillon témoin montrent encore des difficultés à distinguer entre les différentes pièces de monnaie.

Ces résultats pourraient être expliqués par le fait que les arts-plastiques offrent une gamme d'activités manuelles et concrètes qui pourrait aider les élèves à dépasser certaines difficultés. 
En d'autres termes, plusieurs auteurs $[25,26,30,20]$ suggèrent l'importance d'avoir des apprentissages concrets et fonctionnels qui pourraient consolider l'apprentissage de cette population. Par ailleurs, ces élèves ayant une déficience intellectuelle légère souffrent de difficultés au niveau de la mémoire de travail ce qui provoque des difficultés de stockage et de mémorisation; donc il est nécessaire d'adopter des démarches d'apprentissage par le concret qui stimule leurs fonctions exécutives et c'est le cas des activités menées par les artsplastiques. Cependant, la comparaison entre les résultats du pré et du post-test ne montre aucune différence pour les deux échantillons au niveau de la géométrie et l'utilisation de l'unité de centimètre. En fait, les élèves n'arrivent pas à dessiner les formes géométriques demandées (cercle, carré, triangle) en respectant les normes données. Et ceci même après l'intervention avec l'échantillon expérimental par une activité de dessin et de coloriage de formes géométriques. Ces résultats pourraient être expliqués par les difficultés sensorimotrices chez ces élèves ainsi que leurs difficultés pour maitriser l'espace. En outre, l'exécution de cette tache de dessin des formes géométriques nécessitent une mémoire procédurale et une mémoire de travail qui sont limitées chez les personnes porteuses d'une déficience intellectuelle [13]. Une étude statistique pourrait améliorer ces résultats. Les tableaux suivants présentent les résultats obtenus dans le pré et le post-test pour chaque apprenant des deux échantillons.

Tableau 2 : Les résultats du pré et du post-test de l'échantillon expérimental

(MT : Distinguer entre les aiguilles ; MA : Distinguer entre les pièces de monnaie entre 5 millièmes et 5 dinars ; Géométrie : Dessiner des formes géométriques : cercle, carrée et triangle).

\begin{tabular}{|c|c|c|}
\hline Apprenant & Pré-test & Post-test \\
\hline$A 1$ & $\begin{array}{l}\text { MT }^{*}: \text { N'est pas acquis } \\
\text { MA }^{* *}: \text { N'est pas acquis } \\
\text { Géométrie }^{* * *}: \text { N'est pas acquis }\end{array}$ & $\begin{array}{l}\text { MT : Acquis } \\
\text { MA : Acquis } \\
\text { Géométrie : N'est pas acquis }\end{array}$ \\
\hline$A 2$ & $\begin{array}{l}\text { MT : N'est pas acquis } \\
\text { MA : N'est pas acquis } \\
\text { Géométrie : N'est pas acquis }\end{array}$ & $\begin{array}{l}\text { MT : Acquis } \\
\text { MA : Acquis } \\
\text { Géométrie : N'est pas acquis }\end{array}$ \\
\hline$A 3$ & $\begin{array}{l}\text { MT : N'est pas acquis } \\
\text { MA : N'est pas acquis } \\
\text { Géométrie : N'est pas acquis }\end{array}$ & $\begin{array}{l}\text { MT : Acquis } \\
\text { MA : Acquis avec quelques difficultés pour } \\
\text { distinguer entre les valeurs des pièces de } \\
\text { monnaie } \\
\text { Géométrie : N'est pas acquis }\end{array}$ \\
\hline$A 4$ & $\begin{array}{l}\text { MT : Acquis avec difficultés de distinguer } \\
\text { entre les tailles des aiguilles } \\
\text { MA : difficultés pour distinguer entre les } \\
\text { valeurs des pièces de monnaie } \\
\text { Géométrie : N'est pas acquis }\end{array}$ & $\begin{array}{l}\text { MT : Acquis } \\
\text { MA : Acquis } \\
\text { Géométrie : N'est pas acquis }\end{array}$ \\
\hline$A 5$ & $\begin{array}{l}\text { MT : Acquis avec difficultés de distinguer } \\
\text { entre les tailles des aiguilles } \\
\text { MA : difficultés pour distinguer entre les } \\
\text { valeurs des pièces de monnaie } \\
\text { Géométrie : Difficultés pour dessiner la } \\
\text { carrée et le triangle }\end{array}$ & $\begin{array}{l}\text { MT : Acquis } \\
\text { MA : Acquis } \\
\text { Géométrie : Difficultés pour dessiner la } \\
\text { carrée }\end{array}$ \\
\hline$A 6$ & $\begin{array}{l}\text { MT : N'est pas acquis } \\
\text { MA : N'est pas acquis } \\
\text { Géométrie : N'est pas acquis }\end{array}$ & $\begin{array}{l}\text { MT : Acquis } \\
\text { MA : Acquis } \\
\text { Géométrie : N'est pas acquis }\end{array}$ \\
\hline$A 7$ & $\begin{array}{l}\text { MT : N'est pas acquis } \\
\text { MA : difficultés dans la décomposition. } \\
\text { Géométrie : N'est pas acquis }\end{array}$ & $\begin{array}{l}\text { MT : Acquis } \\
\text { MA : Acquis } \\
\text { Géométrie : N'est pas acquis }\end{array}$ \\
\hline$A 8$ & $\begin{array}{l}\text { MT: Acquis avec difficultés de distinguer } \\
\text { entre les tailles des aiguilles }\end{array}$ & $\begin{array}{l}\text { MT : Acquis } \\
\text { MA : difficultés }\end{array}$ \\
\hline
\end{tabular}




\begin{tabular}{|l|l|l|}
\hline MA : difficultés pour distinguer entre les & $\begin{array}{l}\text { Géométrie : Difficultés pour dessiner la } \\
\text { caleurs des pièces de monnaie } \\
\text { Géométrie : Difficultés pour dessiner la } \\
\text { carrée }\end{array}$ & \\
\hline
\end{tabular}

Tableau 3 : Les résultats du pré et du post-test de l'échantillon témoin

(MT : Distinguer entre les aiguilles; MA : Distinguer entre les pièces de monnaie entre 5 millimes et 5 dinars ; Géométrie : Dessiner des formes géométriques : cercle, carrée et triangle).

\begin{tabular}{|c|c|c|}
\hline Apprenant & Pré-test & Post-test \\
\hline B1 & $\begin{array}{l}\text { MT : N'est pas acquis } \\
\text { MA : N'est pas acquis } \\
\text { Géométrie : Difficultés pour dessiner la } \\
\text { carrée }\end{array}$ & $\begin{array}{l}\text { MT : N'est pas acquis } \\
\text { MA : Acquis avec quelques difficultés pour } \\
\text { distinguer entre les valeurs des pièces de } \\
\text { monnaie } \\
\text { Géométrie : Difficultés pour dessiner la } \\
\text { carrée et le triangle }\end{array}$ \\
\hline$B 2$ & $\begin{array}{l}\text { MT : N'est pas acquis } \\
\text { MA : N'est pas acquis } \\
\text { Géométrie : N'est pas acquis }\end{array}$ & $\begin{array}{l}\text { MT : N'est pas acquis } \\
\text { MA : N'est pas acquis } \\
\text { Géométrie : N'est pas acquis }\end{array}$ \\
\hline B3 & $\begin{array}{l}\text { MT : N'est pas acquis } \\
\text { MA : N'est pas acquis } \\
\text { Géométrie : N'est pas acquis }\end{array}$ & $\begin{array}{l}\text { MT : N'est pas acquis } \\
\text { MA : N'est pas acquis } \\
\text { Géométrie : N'est pas acquis }\end{array}$ \\
\hline B4 & $\begin{array}{l}\text { MT : Acquis avec difficultés de } \\
\text { distinguer entre les tailles des aiguilles } \\
\text { MA : difficultés pour distinguer entre les } \\
\text { valeurs des pièces de monnaie } \\
\text { Géométrie : N'est pas acquis }\end{array}$ & $\begin{array}{l}\text { MT : Acquis avec difficultés de distinguer } \\
\text { entre les tailles des aiguilles } \\
\text { MA : Acquis avec quelques difficultés pour } \\
\text { distinguer entre les valeurs des pièces de } \\
\text { monnaie } \\
\text { Géométrie : N'est pas acquis }\end{array}$ \\
\hline B5 & $\begin{array}{l}\text { MT : Acquis avec difficultés de } \\
\text { distinguer entre les tailles des aiguilles } \\
\text { MA : N'est pas acquis } \\
\text { Géométrie : N'est pas acquis }\end{array}$ & $\begin{array}{l}\text { MT : Acquis avec difficultés de distinguer } \\
\text { entre les tailles des aiguilles } \\
\text { MA : N'est pas acquis } \\
\text { Géométrie : N'est pas acquis }\end{array}$ \\
\hline B6 & $\begin{array}{l}\text { MT : N'est pas acquis } \\
\text { MA : N'est pas acquis } \\
\text { Géométrie : N'est pas acquis }\end{array}$ & $\begin{array}{l}\text { MT : N'est pas acquis } \\
\text { MA : N'est pas acquis } \\
\text { Géométrie : N'est pas acquis }\end{array}$ \\
\hline B7 & $\begin{array}{l}\text { MT : N'est pas acquis } \\
\text { MA : N'est pas acquis } \\
\text { Géométrie : N'est pas acquis }\end{array}$ & $\begin{array}{l}\text { MT : N'est pas acquis } \\
\text { MA : N'est pas acquis } \\
\text { Géométrie : N'est pas acquis }\end{array}$ \\
\hline
\end{tabular}

\subsection{La médiation musicale... une forme d'accessibilité cognitive dans l'apprentissage de la mesure du temps}

Les réponses des élèves des deux échantillons au pré-test nous permettent d'identifier les difficultés suivantes : au niveau de la mesure du temps, les élèves ont des difficultés à distinguer entre les mouvements des aiguilles de la montre et des difficultés à distinguer entre les distances longues et courtes au niveau de la géométrie.

Notre intervention musicale s'est déroulée de la manière suivante : deux élèves tournent suivant un modèle de montre dessiné sur le sol. L'un représente la grande aiguille (aguille des minutes) et l'autre représente la petite aiguille (aiguille des heures). Le départ de l'activité commence au moment où on lance la musique. La consigne consiste à tourner en suivant le rythme de mouvement de chaque aiguille et à s'arrêter à la bonne position de l'heure demandée. Par exemple : on demande aux élèves de tourner en suivant le modèle dessiner 
sur le sol et ils vont s'arrêter à $08: 30$. Après cette activité, les élèves de notre échantillon d'étude arrivent à distinguer entre les mouvements des aiguilles de façon correcte malgré les difficultés rencontres par deux élèves pour écrire les minutes (voir tableau 4). Au contraire, les élèves de l'échantillon témoin qui a suivi l'apprentissage du temps à travers des exercices sur des papiers n'ont pas montré une évolution notable. Cette évolution chez les élèves de l'échantillon expérimental s'expliquerait par l'apport cognitif de la musique puisqu'elle garantit un apprentissage qui stimule les fonctions exécutives comme l'attention. En fait, les personnes ayant une déficience intellectuelle légère souffrent d'un problème au niveau de l'attention sélective qu'est un élément fondamental dans le fonctionnement cognitif au cours e l'apprentissage [13]. C'est pour cela, il est très essentiel d'avoir une stimulation de ces fonctions cruciales dans l'apprentissage et c'est le cas de la médiation musicale, puisqu' elle permet de remédier cognitivement à ces fonctions.

Tableau 4 : Les résultats du pré et du post-test de l'échantillon expérimental (MT : Distinguer entre les minutes et les heures; Géométrie : Distinguer entre les distances longues et courtes).

\begin{tabular}{|c|c|c|}
\hline Apprenant & Pré-test & Post-test \\
\hline$A 1$ & $\begin{array}{l}\text { MT }^{*}: \text { N'est pas acquis } \\
\text { Géométrie }^{* *}: \text { N'est pas acquis }\end{array}$ & $\begin{array}{l}\text { MT : Acquis avec des difficultés pour écrire les } \\
\text { minutes } \\
\text { Géométrie : N'est pas acquis }\end{array}$ \\
\hline$A 2$ & $\begin{array}{l}\text { MT }^{*}: \text { N'est pas acquis } \\
\text { Géométrie }^{* *}: \text { N'est pas acquis }\end{array}$ & $\begin{array}{l}\text { MT : Acquis } \\
\text { Géométrie : Acquis avec quelques difficultés } \\
\text { pour distinguer entre les distances de longueurs } \\
\text { proches }\end{array}$ \\
\hline$A 3$ & $\begin{array}{l}\text { MT }^{*}: \text { Difficultés pour déterminer les } \\
\text { minutes }(10: 30 \text { il l'écrit } 10: 6) \\
\text { Géométrie }^{* *}: \text { N'est pas acquis }\end{array}$ & $\begin{array}{l}\text { MT : Acquis } \\
\text { Géométrie : Acquis }\end{array}$ \\
\hline$A 4$ & $\begin{array}{l}\text { MT }^{*} \text { : N'est pas acquis } \\
\text { Géométrie }^{* *}: \text { N'est pas acquis }\end{array}$ & $\begin{array}{l}\text { MT : Acquis avec des difficultés pour écrire les } \\
\text { minutes } \\
\text { Géométrie : Acquis }\end{array}$ \\
\hline$A 5$ & $\begin{array}{l}\text { MT }^{*} \text { : N'est pas acquis } \\
\text { Géométrie*** }^{* *} \text { N'est pas acquis }\end{array}$ & $\begin{array}{l}\text { MT : Acquis avec des difficultés pour écrire les } \\
\text { minutes } \\
\text { Géométrie : N'est pas acquis }\end{array}$ \\
\hline A6 & $\begin{array}{l}\text { MT }^{*} \text { : Difficultés pour écrire les } \\
\text { heures et les minutes }(10: 30 \text {, il l'écrit } \\
106) \\
\text { Géométrie }^{* *}: \text { N'est pas acquis }\end{array}$ & $\begin{array}{l}\text { MT : Acquis } \\
\text { Géométrie : Acquis quelques difficultés pour } \\
\text { distinguer entre les distances de longueurs } \\
\text { proches }\end{array}$ \\
\hline$A 7$ & $\begin{array}{l}\text { MT }{ }^{*}: \text { N'est pas acquis } \\
\text { Géométrie }^{* *}: \text { N'est pas acquis }\end{array}$ & $\begin{array}{l}\text { MT : Acquis } \\
\text { Géométrie : N'est pas acquis }\end{array}$ \\
\hline$A 8$ & $\begin{array}{l}\text { MT }{ }^{*}: \text { N'est pas acquis } \\
\text { Géométrie }^{* *}: \text { N'est pas acquis }\end{array}$ & $\begin{array}{l}\text { MT : Acquis } \\
\text { Géométrie : N'est pas acquis }\end{array}$ \\
\hline
\end{tabular}

Pour la distinction entre les distances, nous avons mis en œuvre une activité musicale qui se base sur la marche en suivant le rythme musical entendu (un rythme accéléré pour les distances longues et un rythme faible pour les courtes distances). Pour les élèves du deuxième échantillon, l'éducatrice met en œuvre des activités de géométrie. La consigne consiste à mesurer la taille des formes géométriques (fig.7). Après l'intervention, les élèves des deux échantillons montrent aussi des difficultés pour apprendre les distances. Nous pouvons avancer que la distinction entre les distances (longue/courte) nécessite beaucoup d'investissement des fonctions cognitives comme l'adaptation et l'orientation spatiale, des apprentissages qui pourraient être absents chez les élèves ayant une déficience intellectuelle légère. Il est à noter que la déficience intellectuelle est toujours associée aux problèmes au niveau des comportements adaptatifs conceptuels, sociaux et pratiques [13] et ceci pourrait 
expliquer la limitation de ces résultats dans le présent cas. Un travail à long terme par le biais des autres médiations et avec un échantillon assez large pourrait montrer des autres résultats.

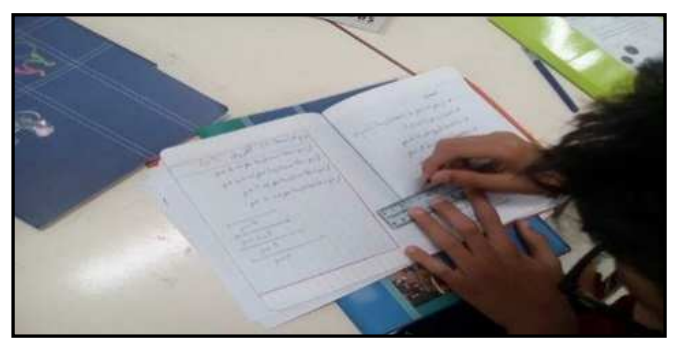

Fig. 7. Apprentissage de la géométrie et de l'unité de centimétre de l'échantllion témoin (ET)

Les résultats pour chaque apprenant de l'échantillon témoin est présenté dans les tableau suivant :

Tableau 5 : Les résultats du pré et du post-test de l'échantillon témoin

(MT : Distinguer entre les minutes et les heures; Géométrie : Distinguer entre les distances longues et courtes).

\begin{tabular}{|c|c|c|}
\hline Apprenant & Pré-test & Post-test \\
\hline B1 & $\begin{array}{l}\text { MT }^{*}: \text { N'est pas acquis } \\
\text { Géométrie }^{* *} \text { : N'est pas acquis }\end{array}$ & $\begin{array}{l}\text { MT : N'est pas acquis } \\
\text { Géométrie : N'est pas acquis }\end{array}$ \\
\hline$B 2$ & $\begin{array}{l}\text { MT }^{*}: \text { N'est pas acquis } \\
\text { Géométrie }^{* *}: \text { N'est pas acquis }\end{array}$ & $\begin{array}{l}\text { MT: Acquis avec des difficultés pour écrire les } \\
\text { minutes } \\
\text { Géométrie : N'est pas acquis }\end{array}$ \\
\hline B3 & $\begin{array}{l}\text { MT }^{*}: \text { N'est pas acquis } \\
\text { Géométrie }^{* *}: \text { N'est pas acquis }\end{array}$ & $\begin{array}{l}\text { MT : N'est pas acquis } \\
\text { Géométrie : N'est pas acquis }\end{array}$ \\
\hline B4 & $\begin{array}{l}\text { MT* }^{*} \text { N'est pas acquis } \\
\text { Géométrie*** }^{* *} \text { N'est pas acquis }\end{array}$ & $\begin{array}{l}\text { MT : N'est pas acquis } \\
\text { Géométrie : N'est pas acquis }\end{array}$ \\
\hline B5 & $\begin{array}{l}\text { MT }^{*} \text { : Difficultés pour écrire les } \\
\text { heures et les minutes }(10: 30 \text {, il } \\
\text { l'écrit 106) } \\
\text { Géométrie }^{* *} \text { : N'est pas acquis }\end{array}$ & $\begin{array}{l}\text { MT : Difficultés pour écrire les heures et les } \\
\text { minutes }(10: 30 \text {, il l'écrit } 106) \\
\text { Géométrie : N'est pas acquis }\end{array}$ \\
\hline B6 & $\begin{array}{l}\text { MT }^{*}: \text { N'est pas acquis } \\
\text { Géométrie }^{* *}: \text { N'est pas acquis }\end{array}$ & $\begin{array}{l}\text { MT : N'est pas acquis } \\
\text { Géométrie : N'est pas acquis }\end{array}$ \\
\hline B7 & $\begin{array}{l}\text { MT : Acquis } \\
\text { Géométrie : N'est pas acquis }\end{array}$ & $\begin{array}{l}\text { MT: Acquis avec des difficultés pour écrire les } \\
\text { minutes } \\
\text { Géométrie : N'est pas acquis }\end{array}$ \\
\hline
\end{tabular}

\subsection{La médiation théâtrale... une forme d'apprentissage motivant}

Pour intervenir sur l'habilité de mesure de temps, nous faisons recours à une activité théâtrale par un jeu de rôles où les élèves vont représenter et lire l'heure sur la montre. A chaque fois, deux élèves doivent jouer l'un le rôle de roi qui va représenter le temps sur la montre et l'autre le rôle d'un citoyen qui va lire l'heure représentée. La comparaison entre les résultats du pré et post-test montrent que l'intervention par la médiation théâtrale a permis aux élèves de cet échantillon d'acquérir une capacité pour distinguer entre les heures et les minutes ainsi que de les lire et de les représenter sur la montre. 

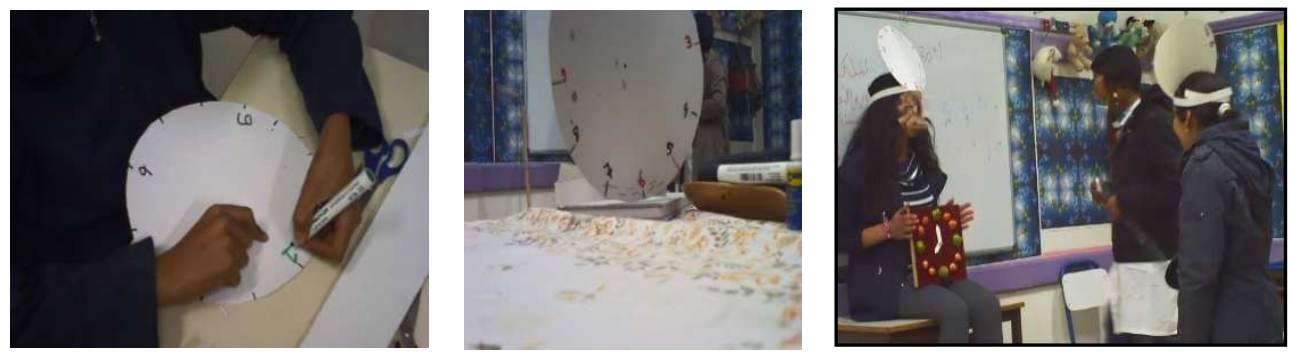

Fig. 8. Apprentissage de mesure du temps par la médiation théâtrale.

En outre, pour la manipulation de l'argent, les élèves jouent le rôle d'un marchand et d'un client. Au cours de cette activité, ils vont apprendre la gestion et la manipulation de l'argent. Une comparaison entre les résultats de pré et post-test nous montrent que les élèves de cet échantillon arrivent à manipuler et à gérer les pièces de monnaie dans des situations de la vie quotidienne. Cette activité a permis aux élèves d'investir leurs expériences dans la vie quotidienne pour apprendre la manipulation de l'argent.
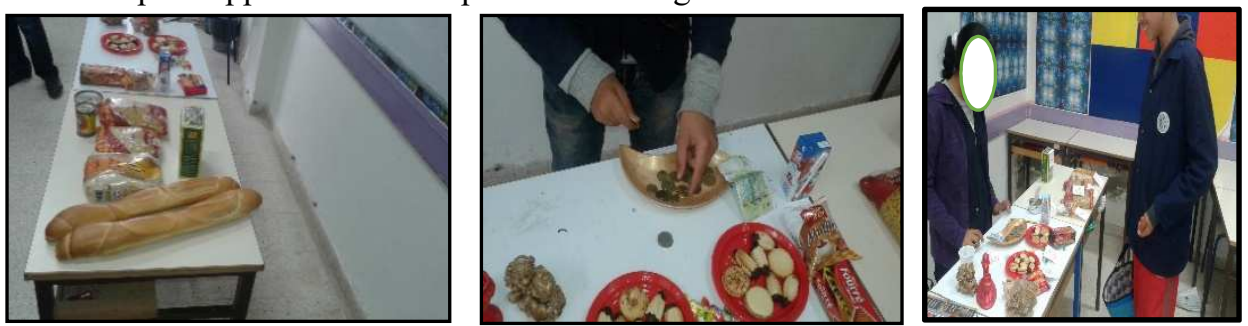

Fig. 9. Apprentissage de la manipulation de l'argent par la médiation théâtrale.

Enfin, concernant l'évaluation des quantités, les élèves montrent des difficultés à distinguer entre les unités et les outils de mesure des poids et des longueurs. Afin d'améliorer cette habilité, nous avons mis en place une activité de jeu de rôle d'un tailleur qui va mesurer la taille d'un tissu demandé par un client et ceci pour la mesure des longueurs. Pour l'habilité de l'évaluation des poids, nous avons mis en place une activité « faire des courses » par les élèves dans l'épicerie de la classe dont laquelle ils vont jouer le rôle d'un vendeur qui va mesurer un poids d'un produit demandé par un client. Au cours de ces activités, les élèves manipulent les différentes unités et outils de mesure des poids et des longueurs et les utilisent dans des situations courantes de la vie quotidienne. Le dépouillement des résultats de posttest montre que les élèves arrivent après l'intervention éducative spécialisée de distinguer entre les différents outils et unités de mesures des poids et des longueurs.

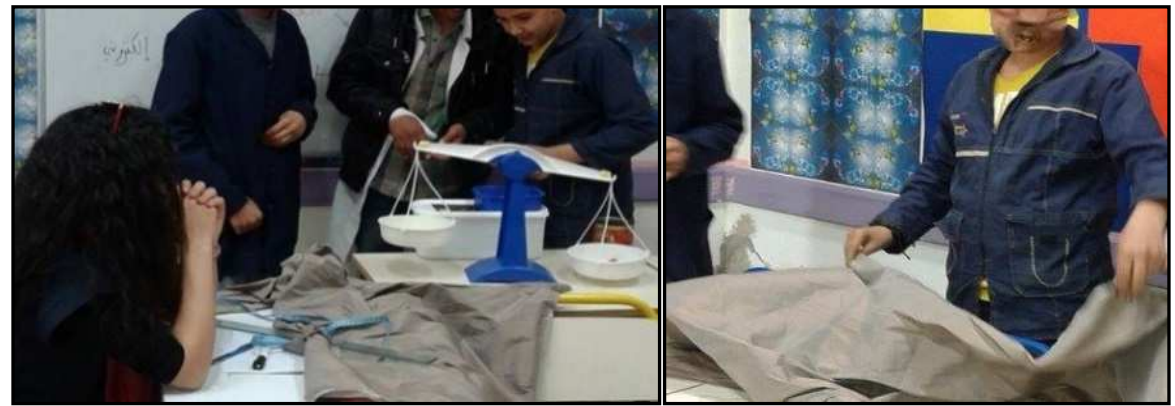

Fig. 10. Apprentissage de l'évaluation des quantités par la médiation théâtrale. 
Ces résultats montrent que la médiation théâtrale pourrait être une forme d'apprentissage motivante puisqu'elle offre des activités similaires et utiles à celles de la vie quotidienne et elle donne un sens aux apprentissages. Dans ce cadre, l'objectif principal de l'enseignement pour les personnes déficients intellectuelles est d'améliorer leur quotidien [27]. Ainsi, les mathématiques sont impliquées dans de nombreuses activités de la vie quotidienne. C'est pour cette raison qu'il est très important de mettre en œuvre des activités en relation avec le quotidien de l'élève. En comparaison avec les autres médiations utilisées, la médiation théâtrale est plus efficace pour l'apprentissage des notions et des habilités mathématiques comme la mesure du temps et la manipulation de l'argent. Dans le tableau 6, nous présentons les résultats du pré et du post-test pour l'échantillon expérimental :

Tableau 6 : Les résultats du pré et du post-test de l'échantillon expérimental

(MT : Lire le temps ; MA : Manipuler l'argent dans des situations de la vie quotidienne ; EQ : Utiliser les outils et les unités de mesure des poids et des longueurs).

\begin{tabular}{|c|c|c|}
\hline Apprenant & Pré-test & Post-test \\
\hline$A 1$ & $\begin{array}{l}\text { MT }^{*}: \text { N'est pas acquis } \\
\text { MA }^{* *}: \text { N'est pas acquis } \\
\text { EQ }^{* * *}: \text { N'est pas acquis }\end{array}$ & $\begin{array}{l}\text { MT : Acquis } \\
\text { MA : Acquis } \\
\text { EQ : Acquis }\end{array}$ \\
\hline$A 2$ & $\begin{array}{l}\text { MT : N'est pas acquis } \\
\text { MA : N'est pas acquis } \\
\text { EQ : N'est pas acquis }\end{array}$ & $\begin{array}{l}\text { MT : Acquis } \\
\text { MA : Acquis } \\
\text { EQ : Acquis }\end{array}$ \\
\hline$A 3$ & $\begin{array}{l}\text { MT : Difficultés pour déterminer les } \\
\text { minutes } \\
\text { MA : N'est pas acquis } \\
\text { EQ : N'est pas acquis }\end{array}$ & $\begin{array}{l}\text { MT: Acquis } \\
\text { MA : Acquis } \\
\text { EQ : Acquis avec quelques difficultés de } \\
\text { déterminer l'unité de mesure des longueurs }\end{array}$ \\
\hline$A 4$ & $\begin{array}{l}\text { MT : N'est pas acquis } \\
\text { MA : N'est pas acquis } \\
\text { EQ : ne distingue pas entre les outils de } \\
\text { mesure des longueurs et des poids }\end{array}$ & $\begin{array}{l}\text { MT : Acquis } \\
\text { MA : Acquis } \\
\text { EQ : Acquis }\end{array}$ \\
\hline$A 5$ & $\begin{array}{l}\text { MT : N'est pas acquis } \\
\text { MA : N'est pas acquis } \\
\text { EQ : Acquis avec difficultés de } \\
\text { connaitre l'outil de mesure des } \\
\text { longueurs }\end{array}$ & $\begin{array}{l}\text { MT : Acquis } \\
\text { MA : Acquis avec des difficultés pour } \\
\text { déterminer la somme totale des pièces de } \\
\text { monnaie. } \\
\text { EQ : Acquis }\end{array}$ \\
\hline A6 & $\begin{array}{l}\text { MT : N'est pas acquis } \\
\text { MA : N'est pas acquis } \\
\text { EQ : N'est pas acquis }\end{array}$ & $\begin{array}{l}\text { MT : Acquis } \\
\text { MA : Acquis } \\
\text { EQ : Acquis }\end{array}$ \\
\hline$A 7$ & $\begin{array}{l}\text { MT : N'est pas acquis } \\
\text { MA : N'est pas acquis } \\
\text { EQ : N'est pas acquis }\end{array}$ & $\begin{array}{l}\text { MT : N'est pas acquis } \\
\text { MA : Acquis } \\
\text { EQ : N'est pas acquis }\end{array}$ \\
\hline$A 8$ & $\begin{array}{l}\text { MT : N'est pas acquis } \\
\text { MA : N'est pas acquis } \\
\text { EQ : N'est pas acquis }\end{array}$ & $\begin{array}{l}\text { MT : Acquis } \\
\text { MA : Acquis } \\
\text { EQ : Acquis }\end{array}$ \\
\hline
\end{tabular}

Pour l'échantillon témoin, nous n'avons pas noté une différence majeure entre les résultats du pré et post-test (voir tableau 7). En d'autres termes, les élèves de cet échantillon montrent de faibles acquisitions des notions et des habilités mathématiques comme la mesure du temps, la manipulation de l'argent ou l'évaluation des quantités. Ceci pourrait être le résultat de l'absence de l'adaptation dans les durées des séquences d'apprentissage dispensées à ces 
élèves par les éducateurs spécialisés ainsi que le non-respect des potentialités et les singularités de chaque élève [43].
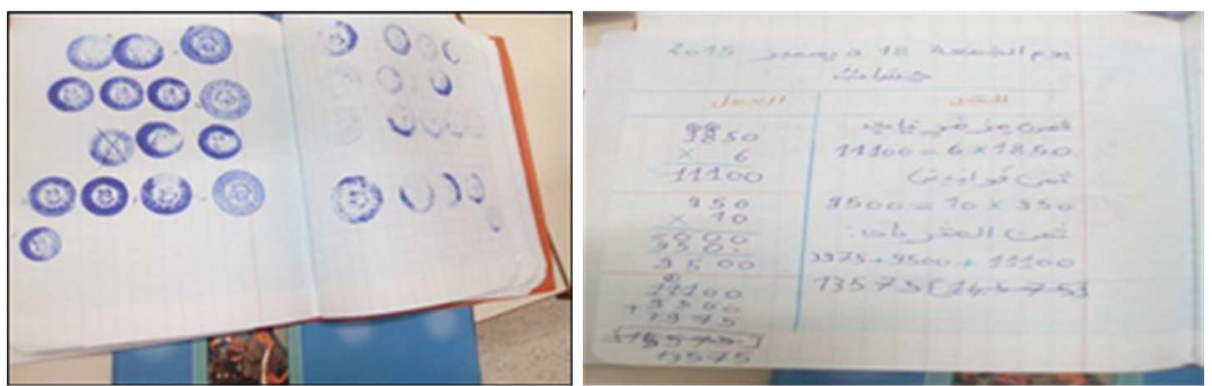

Fig. 11. Apprentissage de la manipulation de l'argent pour l'échantillon témoin.

Tableau 7 : Les résultats du pré et du post-test de l'échantillon témoin

(MT : Lire le temps ; MA : Manipuler l'argent dans des situations de la vie quotidienne ; EQ : Utiliser les outils et les unités de mesure des poids et des longueurs).

\begin{tabular}{|c|c|c|}
\hline Apprenant & Pré-test & Post-test \\
\hline B1 & $\begin{array}{l}\text { MT : N'est pas acquis } \\
\text { MA : N'est pas acquis } \\
\text { EQ : ne distingue pas entre les outils de } \\
\text { mesure des longueurs et des poids }\end{array}$ & $\begin{array}{l}\text { MT : N'est pas acquis } \\
\text { MA : N'est pas acquis } \\
\text { EQ : ne distingue pas entre les outils de mesure } \\
\text { des longueurs et des poids }\end{array}$ \\
\hline$B 2$ & $\begin{array}{l}\text { MT : N'est pas acquis } \\
\text { MA : N'est pas acquis } \\
\text { EQ : N'est pas acquis }\end{array}$ & $\begin{array}{l}\text { MT : N'est pas acquis } \\
\text { MA : N'est pas acquis } \\
\text { EQ : N'est pas acquis }\end{array}$ \\
\hline B3 & $\begin{array}{l}\text { MT : N'est pas acquis } \\
\text { MA : N'est pas acquis } \\
\text { EQ : N'est pas acquis }\end{array}$ & $\begin{array}{l}\text { MT : n'est pas acquis } \\
\text { MA : Acquis } \\
\text { EQ : N'est pas acquis }\end{array}$ \\
\hline B4 & $\begin{array}{l}\text { MT : Difficultés pour déterminer les } \\
\text { minutes } \\
\text { MA : N'est pas acquis } \\
\text { EQ : N'est pas acquis }\end{array}$ & $\begin{array}{l}\text { MT : N'est pas acquis } \\
\text { MA : N'est pas acquis } \\
\text { EQ : N'est pas acquis }\end{array}$ \\
\hline B5 & $\begin{array}{l}\text { MT : Acquis } \\
\text { MA : Acquis } \\
\text { EQ : Acquis }\end{array}$ & $\begin{array}{l}\text { MT : N'est pas acquis } \\
\text { MA : N'est pas acquis } \\
\text { EQ : N'est pas acquis }\end{array}$ \\
\hline B6 & $\begin{array}{l}\text { MT : Difficultés pour déterminer les } \\
\text { minutes } \\
\text { MA : N'est pas acquis } \\
\text { EQ : N'est pas acquis }\end{array}$ & $\begin{array}{l}\text { MT : N'est pas acquis } \\
\text { MA : N'est pas acquis } \\
\text { EQ : N'est pas acquis }\end{array}$ \\
\hline B7 & $\begin{array}{l}\text { MT : N'est pas acquis } \\
\text { MA : N'est pas acquis } \\
\text { EQ : N'est pas acquis }\end{array}$ & $\begin{array}{l}\text { MT : N'est pas acquis } \\
\text { MA : N'est pas acquis } \\
\text { EQ : N'est pas acquis }\end{array}$ \\
\hline
\end{tabular}

\subsection{L'apprentissage par la découverte ... une forme d'apprentissage signifiant}

Le recours à l'apprentissage par la découverte en tant que moyen d'apprentissage [37], permet de mettre en situation des activités de la vie quotidienne qui offrent aux élèves les conditions pour découvrir et pour mobiliser leurs connaissances dans des situations vécues et signifiantes $[24,48]$ : citons l'exemple de l'activité de vendeur et l'activité de tailleur. La réalisation des expériences avec du matériel habituellement disponible dans le quotidien des élèves permet aussi d'enrichir leur apprentissage et ceci dans le cas de l'activité du marchand. 
Toutes les activités sont réalisées afin de guider les élèves à explorer et à découvrir les notions et les habilités mathématiques en manipulant un matériel et en vivant des situations de la vie quotidienne. Les situations de découverte permettent aux élèves non seulement d'apprendre ces savoirs mathématiques mais d'augmenter aussi leur motivation d'apprentissage. Les séances de débat menées après chaque activité nous donnent une idée sur l'évolution des connaissances de ces élèves. Nous pouvons avancer que ce moyen d'apprentissage est signifiant puisqu'il permet aux élèves ainsi de relier leurs connaissances précédentes avec les nouveaux apprentissages dont "le sujet est capable de relier une nouvelle information à ce qu'il sait déjà ; il est de plus capable d'établir des interrelations ou d'imposer une structure aux nouvelles informations et, par conséquent, de réduire les efforts de mémorisation qu'exigerait l'activité d'apprentissage » [45]. Par ailleurs, cette forme d'apprentissage permet aux élèves ayant une déficience intellectuelle légère d'agir de façon autonome dans leur vie quotidienne et professionnelle et ce qui leur permet de s'intégrer dans la société [48].

\section{Conclusion : la médiation artistique est une forme d'accessibilité pédagogique et aux savoirs mathématiques}

Notre recherche a abouti à montrer que la médiation artistique est plus efficace que l'approche pédagogique traditionnelle pour l'apprentissage des élèves ayant une déficience intellectuelle légère de quelques notions et habilités mathématiques qui sont utiles dans la vie quotidienne comme la manipulation de l'argent, la mesure du temps, l'évaluation des quantités (les longueurs et les poids), la géométrie et l'utilisation de l'unité de centimètre pour dessiner des formes géométriques.

Nous avons pu démontrer, dans le cadre de notre étude que cette médiation éducative [46] joue un rôle pour favoriser l'accès aux apprentissages. En d'autres termes, les activités mises en œuvre s'articulent autour de deux formes d'accessibilité : l'accessibilité pédagogique et l'accessibilité aux savoirs. La première est définie comme l'ensemble des adaptations pédagogiques, des pratiques, des savoir-faire et des supports d'aides pour permettre à chacun d'apprendre selon ses potentialités individualisées afin de réduire les situations de handicap au sein même de la classe [7,44]. La mise en place des activités par le biais des médiations artistiques a permis aussi aux élèves d'apprendre chacun à sa façon et à sa manière. En fait, nous adoptons une démarche d'apprentissage en fonction de leur profil intellectuel et éducatif, nous avons fait recours à la théorie des intelligences multiples dont l'intelligence musicale et kinésique afin de leur permettre d'investir leurs capacités, nous avons diversifié aussi les activités éducatives pour les motiver comme nous avons adopté une durée plus longue pour les activités. Par ailleurs, les différentes activités se basent sur l'utilisation du matériel concret inspiré de la vie quotidienne des élèves et ceci leur a permis d'apprendre les notions et les habilités mathématiques à travers des situations similaires à celles de la vie quotidienne. Cependant, les élèves de l'échantillon témoin qui ont reçu ces savoirs par le biais d'une approche pédagogique traditionnelle montrent des difficultés même après l'intervention de l'éducatrice. Ces difficultés pourraient être expliquées par le fait que cette méthode n'offre pas des conditions d'accessibilité et ne respecte pas les besoins individualisés et les singularités de chaque élève. 
L'utilisation de la médiation artistique comme moyen d'apprentissage offre aussi les conditions d'accessibilité aux savoirs. En effet, le recours à cette médiation a permis aux élèves de l'échantillon expérimental d'apprendre des savoirs mathématiques utiles dans la vie quotidienne et professionnelle (puisque ces élèves ont suivi aussi une formation professionnelle en pâtisserie) tels que la manipulation de l'argent, l'évaluation des quantités, la mesure du temps, etc. L'accessibilité aux savoirs ou l'accessibilité didactique est définie comme l'ensemble des conditions qui permettent aux élèves d'accéder à l'étude des savoirs : formes d'étude, situations d'enseignement et d'apprentissage, ressources, accompagnement [6]. Dans ce cadre, nous avons essayé de mettre en œuvre des situations d'enseignement et d'apprentissage qui favorisent l'accès aux savoirs et c'est l'exemple de la médiation théâtrale qui garantit l'apprentissage des savoirs mathématiques à travers la mise en scène des situations similaires à celles de la vie quotidienne. En outre, nous avons pu mettre en évidence, à travers les médiations artistiques, un certain nombre de situations pour rendre possible aux élèves de l'échantillon expérimental d'accéder aux savoirs mathématiques. Ces situations sont : D'une part, des situations en relation avec les dynamiques topogénétiques qui consistent l'ensemble des rapports des topos, les positions et les rôles des différents acteurs dans l'espace didactique [49]. Ces dynamiques sont observées dans ce travail entre les élèves et ceci au cours des activités de théâtre et de musique puisqu'ils arrivent à jouer des rôles différents, d'interagir et de se positionner selon le rôle attribué à chacun d'entre eux pendant l'activité. Cette dynamique topogénétiques est installée aussi entre les élèves et l'éducateur à travers les orientations, les consignes, la prise de position et l'engagement dans l'activité. D'autre part, nous avons mis trois types de régulations $[9,10]$ : tout d'abord, les régulations mésogénétiques qui consistent la création des conditions pour que les élèves puissent faire les tâches par exemple nous créons des conditions similaires à celles de la vie quotidienne pour que les élèves réalisent les tâches demandées au cours de l'activité de marchand. Par la suite, les régulations inter-topogénétiques qui consistent l'ensemble de régulations entre deux topos c'est-à-dire, dans le présent cas, entre les élèves et l'éducateur et ceci dans les séances de débats. Enfin, des régulations intra-topogénétiques, c'est-à-dire entre les élèves, en demandant à chaque élève d'aider son camarade à corriger ses erreurs et c'est le cas dans l'activité de musique pour apprendre la mesure de temps.

Il est fondamental ainsi d'avoir des apprentissages et des enseignements utiles destinés aux personnes avec une déficience intellectuelle afin de leur permettre de participer pleinement et effectivement dans la vie sociale (scolaire, professionnelle, etc.) et d'exercer leurs rôles sociaux $[3,20]$. Par ailleurs, la méthode pédagogique utilisée avec les élèves de l'échantillon témoin ne garantit pas ces conditions d'accessibilité aux savoirs puisqu'ils apprennent les notions et les habilités mathématiques à travers une méthode classique comme l'utilisation des papiers, du tableau...

Notre recherche montre également l'importance aussi de l'apprentissage concret pour les élèves ayant une déficience intellectuelle comme un moyen qui aurait un impact sur le fonctionnement et l'autonomie de la personne dans sa vie quotidienne.

Nous pouvons ajouter qu'un travail sur des échantillons assez larges et dans des institutions d'éducation spécialisée diversifiées pourrais améliorer les résultats obtenus ainsi que le travail sur un temps d'intervention assez large peut améliorer les résultats et les acquisitions chez l'échantillon expérimental. Notre recherche se base sur une analyse 
qualitative des résultats obtenus par contre nous pensons qu'une étude quantitative peut mieux améliorer les résultats obtenus avant et après l'expérimentation.

Enfin, ces résultats alimentent un nouveau questionnement auquel d'autres recherches pourraient répondre : comment peut-on améliorer les pratiques des éducateurs spécialisés avec les personnes ayant une déficience intellectuelle légère ? Quels effets de l'accessibilité pédagogique et celle didactique sur les pratiques pédagogiques en faveur des personnes ayant une déficience intellectuelle?

\section{Bibliographie}

1. M. Crahay, L. Verschaffel, E. De Corte, J. Grégorie, Enseignement et apprentissage des mathématiques. (De Boeck, Paris, 2008).

2. D. C. Geary, Children's mathematical development. Research and practical applications, (American Psychological Association, Washington, 1994).

3. G. Portevin-Serre, Enseigner à des enfants déficients. (Champ social éditions, Nimes, 2016).

4. D.P. Rivera, Mathematics education and students with learning disabilities: introduction to the special series. Journal of Learning Disabilities, 30, 2-19 (1997).

5. C. Mary, S. Schmidt, La spécificité de l'enseignement des mathématiques en adaptation scolaire. Education et francophonie, XXXI (2), 1-12 (2003).

6. T. Assude, J-M. Perez, G. Suau, J. Tambone, Conditions d'accessibilité aux savoirs. Dans J. Zaffran (Dirs.), Accessibilité et handicap. (Presses universitaires de Grenoble, Grenoble 2015).

7. S. Ebersold, La grammaire de l'accessibilité. Education et sociétés, 40 (2), 29-47 (2019).

8. G. Demazure, V. Huys, Adapter l'enseignement : un enjeu pédago-éducatif crucial. Dans G. Demazure \& V. Huys (Dirs.), Enseignement et handicap. (Presses universitaires de Grenoble, Grenoble, 2020).

9. H. Benoit, Ressources numériques, didactisation et accessibilité : un modèle typologique. L'exemple de la littéracie pour les enfants sourds. Eduquer et former, 44, 89-102 (2012).

10. G. Suau, T. Assude, Pratiques inclusives en milieu ordinaire : accessibilité didactique et régulations. Carrefours de l'éducation, 42 (2), 155-169 (2016).

11. H. Kroesbergen, E., H. Van Luit, J. Cora, M. Maas, Effectiveness of Explicit and Constructivist Mathematics Instruction for lowachieving Students in The Netherlands. The Elementary School Journal, 104 (3), 233-25 (2004).

12. American Psychiatric Association (APA), Diagnostic and statistical manual of mental disorders- fifth edition (DSM5). (Elsevier Masson SAS, 2013).

13. W. Buntnix \& al, Déficiences intellectuelles. (INSERM, Paris, 2016).

14. L. Bashash, L. Outhred, S. Bochner, Counting Skills and Number Concepts of Students with Moderate Intellectual Disabilities. International Journal of Disability, Development and Education, 50(3), 325-345 (2003).

15. D. Gauthier \& J-R. Poulin. Analyse didactique d'une situation d'apprentissage coopératif des mathématiques au primaire réalisé dans un contexte d'intégration scolaire. Education et francophonie, XXXI (2), 65-81 (2003). 
16. J-L. Paour. Une conception cognitive et développementale de la déficience intellectuelle. Dans S. Lebovici, R. Diatkine, M. Soulé (Dirs.), Nouveau traité de psychiatre de l'enfant et de l'adolescent. (Presses universitaires de France, Paris, 2004).

17. G. Lemoyne, G. Lessard, Les rencontres singulières entre les élèves présentant des difficultés d'apprentissage en mathématiques et leurs enseignants. Education et francophonie, XXXI (2), 13-44 (2003).

18. J. Giroux. Étude des rapports enseignement/apprentissage des mathématiques dans le contexte de l'adaptation scolaire : Problématique et repères didactiques. Education \& didactique, 7(1), 59-86 (2013).

19. C. Hord, E.C. Bouck. Review of Academic Mathematics Instruction for Students with Mild Intellectual Disability. Education and Training in Autism and Developmental Disabilities, 47(3), 389-400 (2012).

20. V. Côté, C. Couture, S. Lippé, Fonctionnement de l'enfant qui présente une déficience intellectuelle et pistes d'interventions. Revue québécoise de psychologie, 37 (2), 121140 (2016).

21. J. Bouchand, L'intégration sociale et professionnelle des personnes avec déficience intellectuelle : la place centrale des compétences adaptatives. La nouvelle revue de l'adaptation et de la scolarisation, 63 (3), 167-184 (2013).

22. B. Céleste, Notes d'histoire sur un concept et des pratiques : déficience intellectuelle. Contraste, 22-23 (1-2), 81-97 (2005)

23. A. Charron \& C. Raby, Synthèse sur le socioconstructivisme. In. C. Raby \& S.Viola (Sous la direction de). Modèles d'enseignement et théories d'apprentissage - de la pratique à la théorie, (Les Éditions CEC, Anjou, 2016)

24. C. Brankaer, P. Ghesquière, B. de Smedt, Numerical magnitude processing in children with mild intellectual disabilities. Research in Developmental Disabilities, 32(6), 28532859 (2011).

25. Ionescu, L'intervention en déficience mentale. (Mardaga, Bruxelles, 1990).

26. J.-L. Lambert, Enseignement spécial et handicap mental. (Mardaga, Bruxelles, 1986).

27. G. Bussy, Déficients intellectuels : comment les aider. Bulletin d'information du CREA Bourgogne, 338 (1), 7-13 (2014).

28. E.H. Kroesbergen, J. E. H. Van Luit, Constructivist mathematics education for students with mild mental retardation. European Journal of Special Needs Education, 20(1), 107116 (2005).

29. J-M. Lesain-Delabarre, M. Colignon. Art-thérapie, médiations artistiques : quelles différences pour quels enjeux ? La nouvelle revue de l'adaptation et de la scolarisation, 72 (4), 295-315 (2015).

30. J. Bala, Methods of Teaching Exceptional Children. (Discovery Publishing House, New Delhi, 2006).

31. A. Brun, Médiations thérapeutiques et psychose infantile. (Dunod, Paris, 2007).

32. S. Korff-Sausse, Art et handicap. (ERES, Toulouse, 2012).

33. M. Colignon, De l'art thérapie à la médiation artistique : quels professionnels pour quels pratiques. (ERES, Toulouse, 2015).

34. E. Lecourt \& T. Schauder. Les art-thérapies : présentation. Dans E. Lecourt \& T. Schauder (Dirs.), Les art-thérapies. (Armand Colin, Malakoff, 2017).

35. E. Lecourt. Musicothérapie. Dans E. Lecourt \& T. Schauder (Dirs.), Les art-thérapies. Malakoff : Armand Colin. 2017. 
36. J. S. Bruner, Quelques éléments de la découverte. Dans L. S. Shulman \& E. R. Keislar (Dirs.), La pédagogie par la découverte. (Éditions Viette, Paris, 1973).

37. G. Thésée, Apprentissage par la découverte. DansC. Raby \& S.Viola (Dirs.), Modèles d'enseignement et théories d'apprentissage - de la pratique à la théorie. (Les Éditions CEC, Anjou, 2016).

38. R. Glaser, Les variables de l'apprentissage par la découverte. Dans L. S. Shulman \& E. R. Keislar (Dirs.), La pédagogie par la découverte. (Éditions Viette, Paris, 1973).

39. M. Thouin, La didactique des sciences de la nature au primaire. (Éditions MultiMondes, Sainte-Foy, 1997).

40. M. Altet, Les pédagogies de l'apprentissage. (PUF, Paris, 2013).

41. M. Bru, Les méthodes en pédagogie. Que suis-je ? (PUF, Paris, 2015).

42. J-P. Resweber, Les pédagogies nouvelles. Que suis-je ? (PUF, Paris, 2017).

43. K. Lamouchi Chebbi, Pratiques de classe en éducation spécialisée Projet individualisé ou improvisation pédagogique ? Dans F. Reichhart, A. Célestin, L. Myazhiom, Z. Rachedi \& M. Mercier (Dirs.), Au carrefour de l'altérité : pratiques et représentations $d u$ handicap dans l'espace francophone. (Presses universitaires de Namur, Namur, 2020).

44. H. Benoit, J. Sagot, L'apport des aides techniques à la scolarisation des élèves handicapés. La nouvelle revue de l'adaptation et de la scolarisation, 43 (3), 19-26 (2008).

45. F. Smith, La compréhension et l'apprentissage : un cadre de référence pour l'enseignement. (Les Éditions HRW, Montréal, 1979).

46. C. Pittet, Médiation artistique. Dans A. Vandevelde-Rougale, P. Fugier, V. de Gaulejac (Dirs.), Dictionnaire de sociologie clinique. (ERES, Toulouse, 2019). 414-415.

47. S. Schnepel, H. Krähenmann, R.-S. Dessemontet, E, Moser, The mathematical progress of students with an intellectual disability in inclusive classrooms: results of a longitudinal study. Mathematics Education Research Journal, 32, 103-119 (2002).

48. A. J. Baroody, The development of basic counting, number, and arithmetic knowledge among children classified as mentally handicapped. International Review of Research in Mental Retardation, 22(22), 51-103 (1999).

49. T. Assude, J-M. Perez, G. Suau, J. Tambone, A. Vérillon, Accessibilité didactique et dynamique topogénétique: une étude de cas. Recherches en Didactique des Mathematiques, 34 (1), 33-57 (2014). 